\title{
Split Mouth De-epithelization Techniques for Gingival Hyperpigmentation for Esthetic Purposes: A Case Series and Review of Literature
}

\author{
Avnika Garg ${ }^{1}$, Baljeet Singh ${ }^{2}$, Alka Sharma ${ }^{3}$
}

\begin{abstract}
A good pink smile is desirable by all age groups, and it can be of concern especially when the gingiva is seen, as in cases with a gummy smile. Thus, melanin hyperpigmentation on gingiva becomes an esthetic problem in individuals when it is present on the facial aspect of the gingiva and is visible during smile and speech. Various depigmentation techniques are available for treatment of gingival hyperpigmentation for this esthetic purpose. Here, the present study clinically assesses the outcome of four different surgical techniques (using scalpel surgery, gingival abrasion, electrosurgery, and lasers) in the same patients and evaluates the reappearance of the pigmentation in the 6-month follow-up period and compares the results.

Keywords: Depigmentation, Melanin, Repigmentation.

International Journal of Experimental Dental Science (2019): 10.5005/jp-journals-10029-1195
\end{abstract}

\section{INTRODUCTION}

An attractive smile has always been the focal point of a person's attention, to improve esthetic appearance and thus self-esteem. It is not only the contrasts of shape, color of teeth, line, and texture that enable us to determine a beautiful but also the gingival tissue is esthetically important. ${ }^{1}$

The color of the gingiva is determined by various factors which include the number and size of blood vessels, thickness of epithelium, quantity of keratinization, and pigments present within the epithelium. The main pigments that contribute to the normal color of the oral mucosa are melanin, carotene, reduced hemoglobin, and oxyhemoglobin. Frequently, gingival hyperpigmentation is caused by excessive melanin deposition in the basal and suprabasal cell layers of the epithelium. Melanin is produced by specific cells known as melanocytes that reside in the basal layer and are then transferred to the basal cells where they are stored in the form of melanosomes. They can also be found in keratinocytes of gingival epithelium. ${ }^{2}$ Melanin pigmentation of the oral mucosa has both diagnostic and anthropologic significance. ${ }^{3}$ Physiological melanin pigmentation of the oral mucosa with variable amount is clinically manifested as multifocal or diffuse pigmentation in different ethnic groups. Although melanin hyperpigmentation of gingiva is usually not a medical problem, patients with "gummy smile" or excessive display while smiling or talking consider their black gums to be unpleasing. ${ }^{4,5}$

Gingival depigmentation, a periodontal plastic surgical procedure, removes gingival hyperpigmentation using various techniques. The only foremost indication for depigmentation therapy is the demand for improved esthetics by a patient. ${ }^{4}$ Various methods have been used for cosmetic therapy of gingival melanin depigmentation where each technique has its own advantages and disadvantages. These include a scraping technique by using a scalpel, abrasion with diamond bur, gingivectomy with or without free gingival autografts, acellular dermal matrix allografts, electrosurgery, chemical cryosurgery, and light amplification by stimulated emission of radiation (LASER). ${ }^{1,2,4}$

\begin{abstract}
${ }^{1}$ Department of Periodontology, Himachal Dental College, Sundernagar, Himachal Pradesh, India

${ }^{2}$ Department of Periodontology, Bhojia Dental College and Hospital, Baddi, Himachal Pradesh, India

${ }^{3}$ ECHS, Allahabad, Uttar Pradesh, India

Corresponding Author: Avnika Garg, Department of Periodontology, Himachal Dental College, Sundernagar, Himachal Pradesh, India, Phone: +91 9915629767, e-mail: avnika.garg@gmail.com

How to cite this article: Garg A, Singh B, Sharma A. Split Mouth Deepithelization Techniques for Gingival Hyperpigmentation for Esthetic Purposes: A Case Series and Review of Literature. Int J Experiment Dent Sci 2019;8(1):6-10.
\end{abstract}

Source of support: Nil

Conflict of interest: None

Electrosurgery in dentistry has been in use since 1914, in which there is a precise application of heat to the soft tissue site to be cut by electrodes. Two types are available: monopolar terminal unit and bipolar terminal unit. In the monopolar unit, current begins with the device and travels along a wire to the oral site and then to an indifferent plate placed behind the patient's back; as the surgical electrode contacts the oral soft tissues, heat is produced and controlled cutting is achieved. Current used in oral electrosurgery is fully rectified and filtered. The advantages of this method are immediate hemostasis, electrode can be bent to the desired clinical need, ease of use, consistent cutting, minimal trauma, and less discomfort. Its disadvantages include fear of use, burning flesh odor, low tactile sensitivity, fear of bone damage, disruption in the action of pacemakers, and poor postoperative healing. ${ }^{6}$

A scalpel technique is one of the first techniques employed for gingival melanin depigmentation that involves surgical removal of gingival epithelium along with a layer of the underlying connective tissue, thus allowing the denuded connective tissue to heal by secondary intention. The new epithelium that forms is devoid of melanin pigmentation. ${ }^{7}$ 
Abrasion is done with the help of a large round diamond bur using minimal pressure and light brushing strokes; however, it needs copious amounts of irrigation. It can be used in conjunction with the scalpel technique. ${ }^{6}$ However, it is difficult to control the depth of de-epithelialization and to obtain adequate access. ${ }^{8}$

Selective ablation of the melanocytes can be done by using a laser beam at a wavelength which specifically absorbs melanin effectively and, thus, destroys the pigmented cells without damaging the nonpigmented epithelial cells at the basal layer of the treated area. Thus, radiation energy is transformed into ablation energy, resulting in cellular rupture and vaporization with minimal heating of the surrounding tissue. ${ }^{3}$

Gingival repigmentation refers to the reappearance of melanin pigmentation after sometime of performing depigmentation procedure which can be assessed clinically and histologically. ${ }^{9}$ Pigment recurrence may occur anywhere from 24 days to 8 years and starts with migration of melanocytes from the adjacent free gingiva. Thus, it becomes a common concern in the treatment of gingival hyperpigmentation. However, the extent and time interval of recurrence varies with different treatment modalities used and the duration of follow-up. ${ }^{1}$

The literature regarding comparison studies by different techniques in same patient is limited. Hence, this study was designed to clinically assess the outcome of four different surgical techniques (using scalpel surgery, gingival abrasion, electrosurgery, and lasers) in the same patient and evaluate the reappearance of the pigmentation in the 6-month follow-up period and compare the results.

\section{Materials and Methods}

The study included six patients, all males, aged 20-30 years, nonsmokers, who visited the Department of Periodontology, Bhojia Dental College, Baddi, Himachal Pradesh, India, in 2013 with the chief complaint of "dark black gums" and, thus, decreased confidence and embarrassment within their peer groups. Inclusion criterion was the presence of moderate to severe melanin pigmentation of the gingiva. Exclusion criteria included systemic diseases that could be associated with healing disturbances (like uncontrolled diabetes and autoimmune diseases) or with gingival hyperpigmentation, pregnancy, lactation, etc. Color density of the gingival pigmentation was assessed using criteria given in Table $1 .^{10}$ Three patients had third degree of pigmentation, and the rest three had second degree of pigmentation.

The history of all patients revealed that the blackish discoloration of gingiva was present since birth, which is suggestive of physiological melanin pigmentation. A clinical examination revealed healthy periodontium with the presence of generalized

Table 1: Gingival pigmentation index

\begin{tabular}{ll}
\hline Degree & Criteria \\
\hline 0 & $\begin{array}{l}\text { Presence of pink color of the gingiva } \\
\text { Absence of any brown or black pigmentation }\end{array}$ \\
1 & $\begin{array}{l}\text { Presence of spots of brown to black pigmentation on } \\
\text { gingiva }\end{array}$ \\
2 & $\begin{array}{l}\text { Presence of brown to black pigmentation on gingiva, } \\
\text { which are more than spots but not diffuse (patches of } \\
\text { pigmentation) } \\
\text { Presence of diffuse brown to black pigmentation involving } \\
\text { papillary, marginal, and attached gingiva }\end{array}$ \\
\hline
\end{tabular}

diffuse melanin pigmentation of gingiva in the mandible and maxilla. Patients were made educated of the nature of physiological hyperpigmentation and were made understood that this phenomenon is not a medical problem and had no influence on their systemic or oral health. The excessive contrast that can result between the skin and the gingival color after the procedure was also explained to the patient. The patients were made fully aware of the clinical procedure and the consequences in detail. A complete medical history and blood investigation was conducted before the procedure, in which it was found that their medical history was noncontributory and the patients were in good health. Preoperative photographs revealing the amount of hyperpigmented gingival display were taken (Fig. 1). Informed consent was obtained from each patient prior to treatment. Considering the patient's concern, a split mouth surgical de-epithelization procedure was decided. A combination of scalpel de-epithelization, bur abrasion, electrosurgery, and laser was planned.

\section{Treatment Protocol}

The following sequence was observed:

- Recording a thorough case history to rule out systemic factors for gingival hyperpigmentation.

- Patient education.

- Oral prophylaxis.

- Surgical depigmentation procedure.

\section{Method}

Local infiltration was given in the concerned area. Care was taken to see that the underlying bone is not exposed. Bleeding was controlled using moist sterile gauze as a pressure pack. All the remnants of the melanin pigment or pigmented areas of the epithelium within the smile zone were completely removed.

\section{Electrosurgery}

About $2 \mathrm{~mL}$ of local anesthesia (lignocaine with adrenaline) was administered as nerve block and/or infiltration in the first quadrant of mouth. A needle electrode was used for incisions, whereas ball electrodes of different diameters were used to coagulate. The advantage of minimal bleeding with a clean field increased the efficacy of the work. The pigmented area in the anterior region

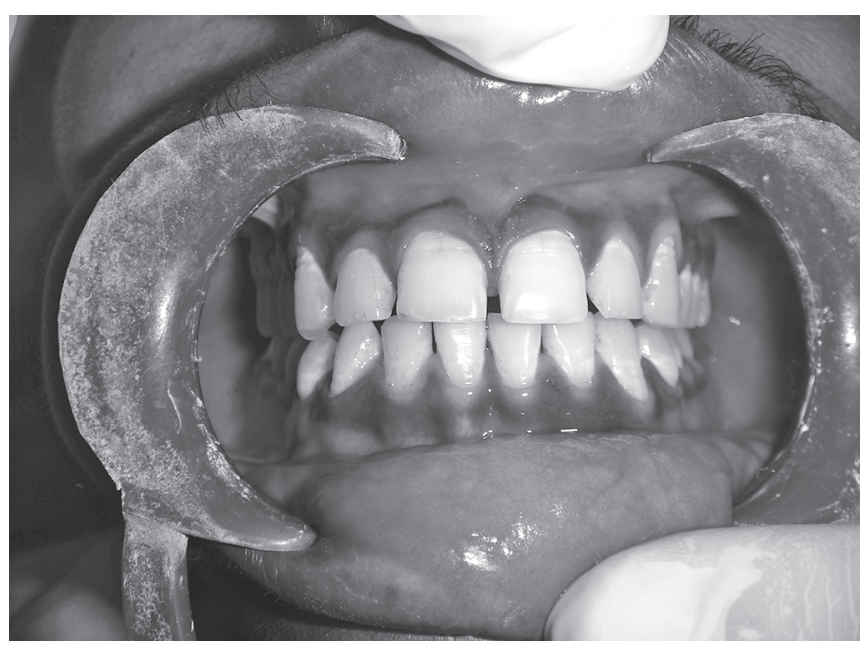

Fig. 1:Preoperative photograph revealing the amount of hyperpigmented gingival display 
destined to undergo electrosurgery de-epithelization was marked using the needle electrode (Fig. 2). Light brushing strokes were used with a loop electrode, and the tip was kept moving all the time, to remove the epithelium (Fig. 3). A prolonged or repeated application of electrode to the tissues was avoided as it could induce heat accumulation and, thus, cause undesired tissue destruction. Moreover, enough care was taken to avoid contact of current with the periosteum and vital teeth.

\section{Surgical Scalpel Procedure}

About $2 \mathrm{~mL}$ of local anesthesia (lignocaine with adrenaline) was administered as nerve block and/or infiltration in the second quadrant of mouth. The pigmented area in the anterior region destined to undergo scalpel de-epithelization was marked using a \#15 scalpel blade (Fig. 4). A split thickness flap was raised and then excised, maintaining the normal architecture of the gingiva. Bleeding was controlled using sterile gauze as a pressure pack.

\section{Surgical Bur Abrasion}

About $2 \mathrm{~mL}$ of local anesthesia (lignocaine with adrenaline) was administered as nerve block and/or infiltration in the third quadrant

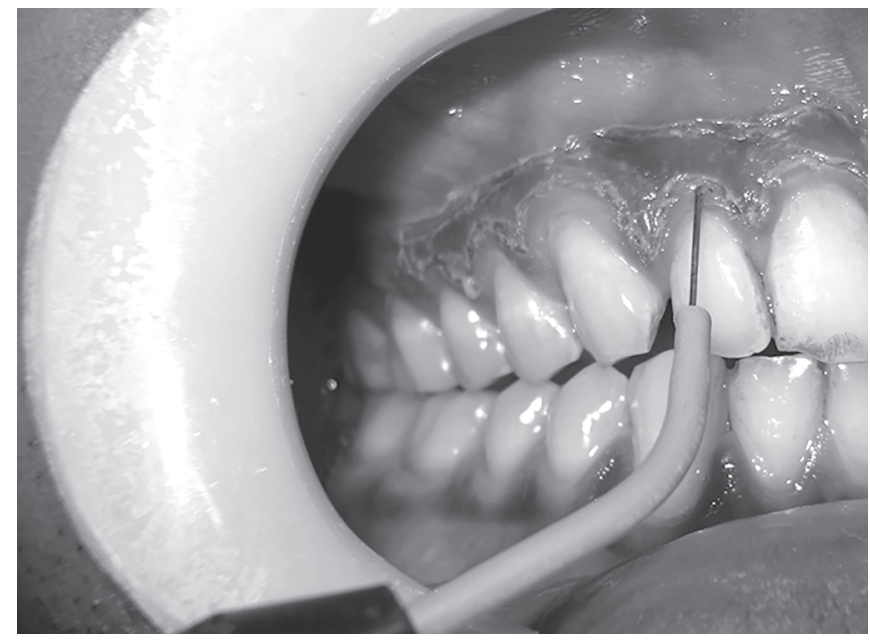

Fig. 2: Area destined to undergo electrosurgery de-epithelization was marked using needle electrode

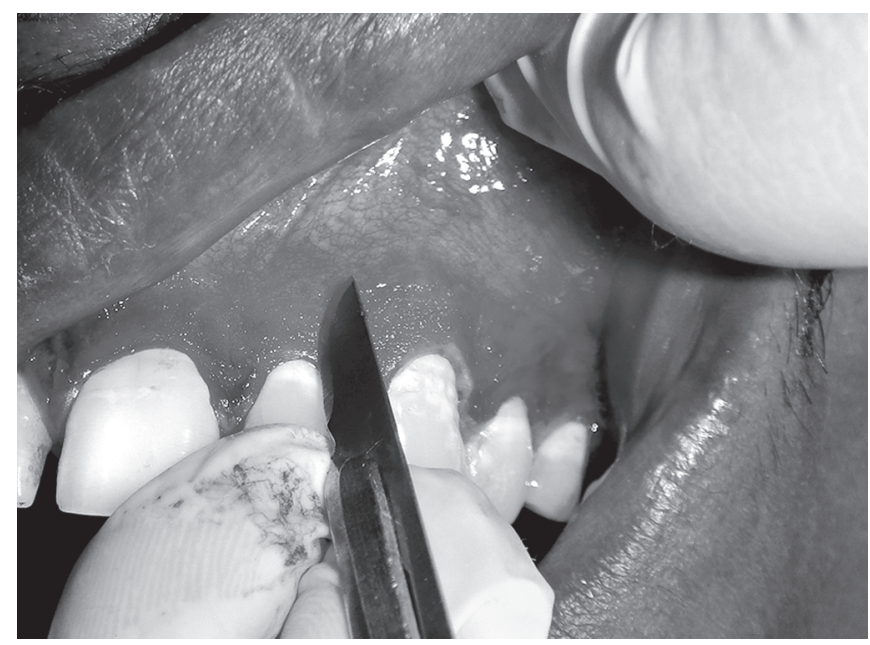

Fig. 4: De-epithelization done using a \#15 scalpel blade of mouth. The pigmented area in the anterior region destined to undergo bur abrasion de-epithelization was marked using a \#15 scalpel blade. Featherlight strokes were used with diamond burs (straight and round shaped) secured in contra-angled micromotor handpiece, and burs were kept moving all the time during the removal of epithelium, without holding the bur in one place (Fig. 5). Sterile saline-soaked gauze was placed on the recipient site as a pressure pack to control bleeding.

\section{LASER}

About $2 \mathrm{~mL}$ of local anesthesia (lignocaine with adrenaline) was administered as nerve block and/or infiltration in the fourth quadrant of mouth. The pigmented area in the anterior region destined to undergo laser de-epithelization was marked using a \#15 scalpel blade. Then, diode LASER beam at $0.7 \mathrm{~W}$, intermittent mode, operating at $980 \mathrm{~mm}$ wavelength with tip of $0.6 \mathrm{~mm}$ diameter, was used in light brushing strokes to remove epithelium (Fig. 6). Lasing time was different according to the degree of pigmentation, the pigmented surface treated, and epithelial thickness. There was no bleeding, and a clean working field was obtained. However, the upper surface of the connective tissue bed got charred and

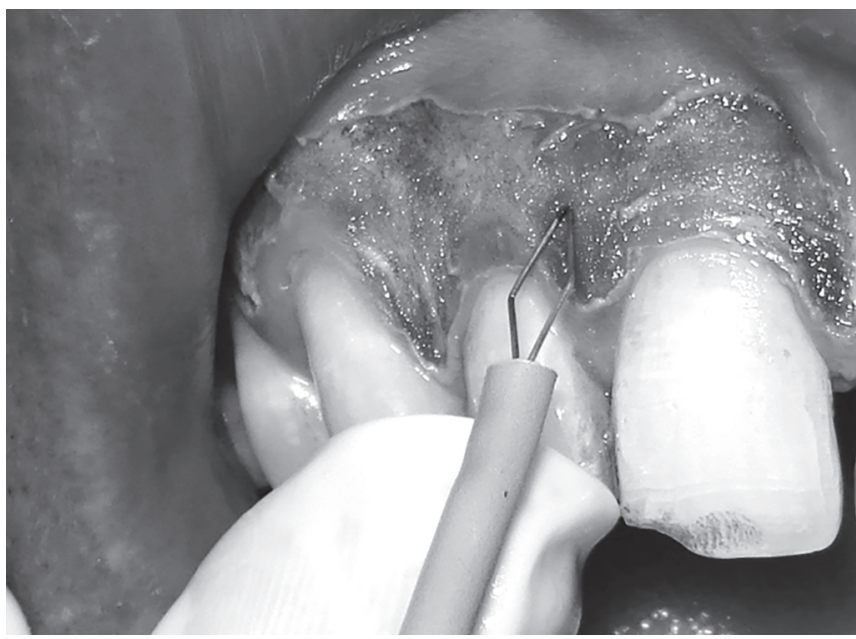

Fig. 3: Use of light brushing strokes with loop electrode to remove the epithelium

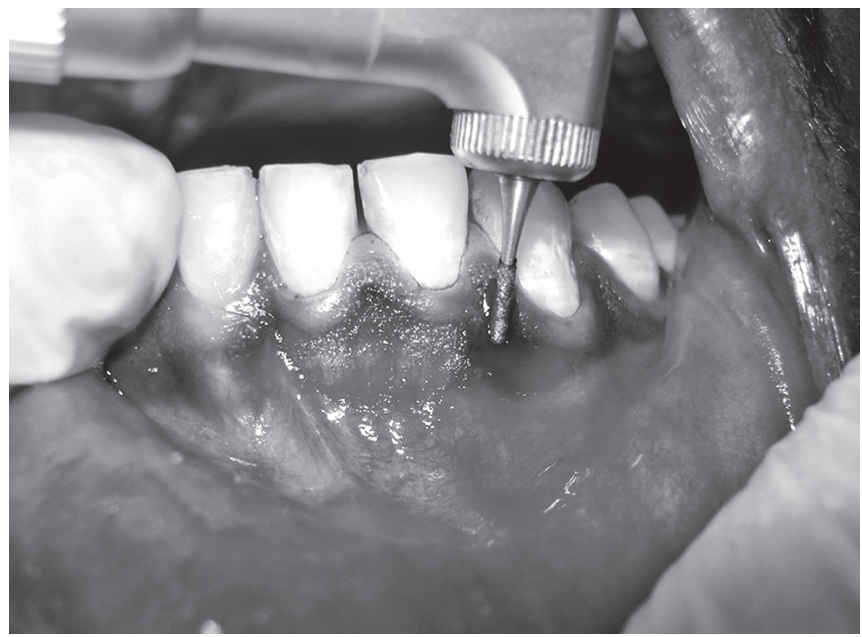

Fig. 5: De-epithelization done by bur abrasion method using diamond burs (straight and round shaped) 


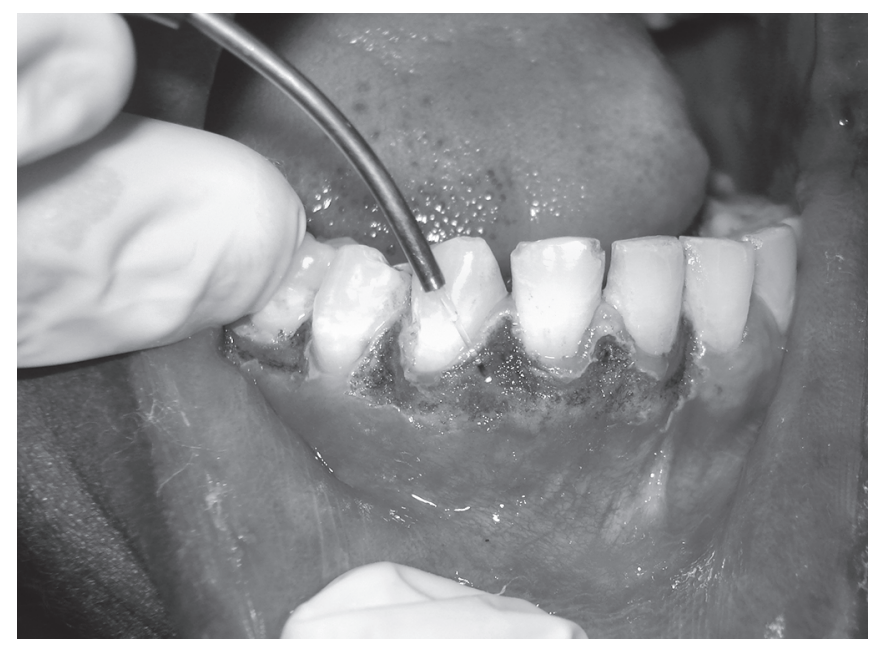

Fig. 6: De-epithelization done using diode LASER beam

appeared black, due to which the underlying pigmented area was difficult to be distinguished.

For all the patients, the exposed depigmented surface was covered with periodontal dressing (Coe-Pak) for 1 week. The patients were prescribed aceclofenac-paracetamol combination B.D. for 3 days, $0.2 \%$ chlorhexidine digluconate rinses twice a day for 2 weeks, and postoperative instructions were given. They were instructed to avoid brushing, alcohol, and spicy food and were advised not to traumatize the surgical area until periodontal dressing was not removed. After 7 days postoperatively, the periodontal dressing was removed, and the surgical area was examined.

\section{Results}

The patients were observed after 1 day, 7 days, 1 month, and 6 months postoperatively. During the first week, superficial necrosis was found at the treated site and a whitish slough separated from the underlying tissue, hence leaving a clean granulating surface. After 2 weeks, it was covered by epithelium, and keratinization was complete at the end of fourth week. However, these findings were based on the clinical observations only as no histologic procedures could be performed to confirm the results. These procedures produced successful results with good patient's satisfaction and a pleasing smile (Fig. 7). Some amount of residual pigmentation was observed in some patients, but because it did not interfere with the patient's smile or esthetics, and as the patients were not keen on its removal, no additional procedure was undertaken. No repigmentation occurred for the initial 6 months, and the patients are being monitored for longitudinal period for any repigmentation.

\section{Discussion}

All the procedures were accepted happily, and the results were excellent as perceived by the patients. However, mild postoperative burning was observed in patients on the first quadrant which was treated using electrosurgery, although no postoperative hemorrhage and infection occurred. Patients kept saying that this procedure was better as it took less time, but there was a mild discomfort after 1-day postoperative. Although electrosurgery had advantages of minimal bleeding and, thus, a cleaner work field, it required more expertise and caution. However, in the

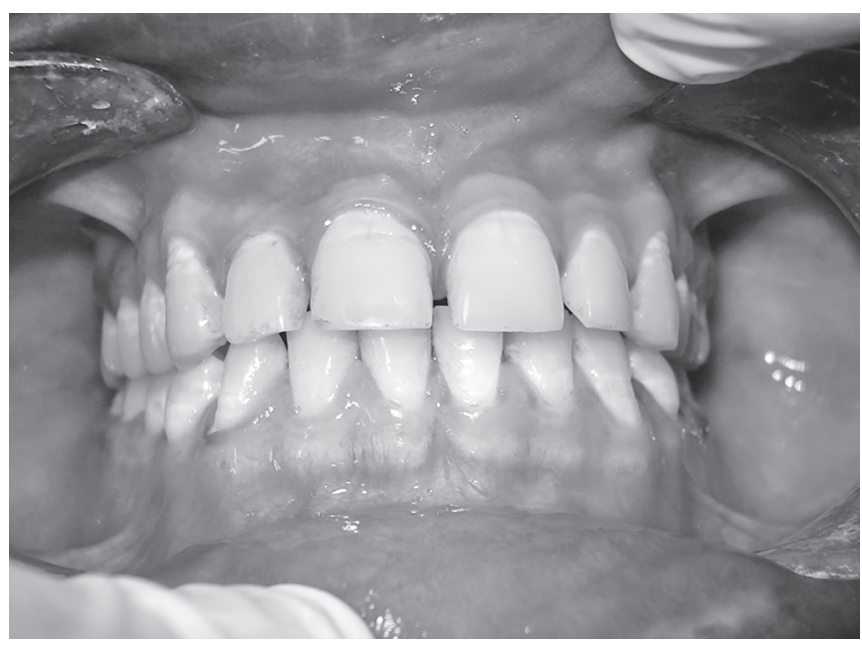

Fig. 7: Successful results with good patient's satisfaction and a pleasant smile

second quadrant which was done using scalpel technique, there was no postoperative pain, hemorrhage, infection, or scarring, and healing was uneventful. The results were excellent and in fact better than electrosurgery which was also supported by the patients' feedbacks. In the third quadrant where bur abrasion was used, the results were similar and comparable with the scalpel technique although patient's acceptance of the procedure was not as good as scalpel technique. Finally, although no postoperative hemorrhage and infection occurred with LASER, there was a delay in healing after 1 week. Moreover, they required sophisticated equipment, occupied large space, and were expensive.

Hence, different techniques for the treatment of pigmented gingiva have been documented, but no technique has shown superiority over the other. So, the selection of a technique should be rather based on clinical experience, thickness of gingival biotype, and the individual's preferences. Moreover, the removal of gingival melanin pigmentation should be performed with caution as inappropriate application of any depigmentation technique may cause delayed wound healing, loss of enamel, gingival recession, damage to underlying periosteum, and bone; thus, adjacent teeth should be protected.

\section{Conclusion}

Gingival melanin pigmentations can occur as a consequence of various local, systemic, environmental, or genetic factors. Hence, to ensure treatment success, the potential causative or aggravating agent of the pigmentation should be identified and eliminated, and if possible, before the surgical treatment for best results. Various depigmentation therapies are available, and every technique has their own merits and demerits; so, the choice of the technique depends on individual preference, clinical expertise, and patient affordability. However, more data are required on comparative techniques to ensure the long-term predictability and success.

\section{References}

1. Mani A, Mani S, Shah S, et al. Management of gingival hyperpigmentation using surgical blade, diamond bur and diode laser therapy: a case report. J Oral Laser Appl 2009;9(4):227-232.

2. Berk G, Atici K, Berk N. Treatment of gingival pigmentation with Er,Cr:YSGG laser. J Oral Laser Appl 2005;5(4):249-253. 
3. Tal H, Oegiesser D, Tal M. Gingival depigmentation by erbium:yag laser: clinical observations and patient responses. J Periodontol 2003;74(11):1660-1667. DOI: 10.1902/jop.2003.74.11.1660.

4. Prasad SSV, Agrawal N, Reddy NR. Gingival depigmentation: a case report. People's Journal of Scientific Research 2010;3(1):27-29.

5. Kumar S, Bhat GS, Bhat KM. Development in techniques for gingival depigmentation- an update. Indian J Dent 2012;3(4):213-221. DOI: 10.1016/j.ijd.2012.05.007.

6. Bhatsange AG, Japati S. Black to pink: clinical evaluation of two different surgical approaches for the treatment of hyperpigmentation. Int J Periodontics Restorative Dent 2011;1(2):136-139.

7. Lawande SA. Gingival melanin depigmentation as an effective treatment for enhancing esthetics: a case report and literature review. JPBMS 2012;22(22):1-4.

8. Atsawasuwan P, Greethong K, Nimmanon V. Treatment of gingival hyperpigmentation for esthetic purposes by $\mathrm{Nd}$ :Yag laser: report of 4 cases. J Periodontol 2000;71(2):315-321. DOI: 10.1902/jop. 2000.71.2.315.

9. Hegde R, Padhye A, Sumanth S, et al. Comparison of surgical stripping; erbium-doped:yttrium, aluminum, and garnet laser; and carbon dioxide laser techniques for gingival depigmentation: a clinical and histologic study. J Periodontol 2013;84(6):738-748. DOI: 10.1902/ jop.2012.120094.

10. Singh V, Giliyar SB, Kumar S, et al. Comparative evaluation of gingival depigmentation by diode laser and cryosurgery using tetrafluoroethane: 18-month follow-up. Clin Adv Periodont 2012;2(3):129-134. DOI: 10.1902/cap.2012.110008.

11. Ghoreishian M, Gheisari R, Fayazi M. Tissue adhesive and suturing for closure of the surgical wound after removal of impacted mandibular 3rd molar. Oral Surg Oral Med Oral Pathol Oral Radiol Endod 2009;108(1):e14-e16. DOI: 10.1016/j.tripleo.2009. 03.001 . 